\title{
Label-free photoacoustic nanoscopy
}

Amos Danielli

Konstantin Maslov

Alejandro Garcia-Uribe

Amy M. Winkler

Chiye $\mathrm{Li}$

Lidai Wang

Yun Chen

Gerald W. Dorn, II

Lihong V. Wang

\section{SPIE.}




\title{
Label-free photoacoustic nanoscopy
}

\author{
Amos Danielli, ${ }^{a}$ Konstantin Maslov, ${ }^{a}$ Alejandro Garcia-Uribe, ${ }^{a}$ Amy M. Winkler, ${ }^{a}$ Chiye $\mathrm{Li}^{\mathrm{a}}{ }^{\mathrm{a}}$ \\ Lidai Wang, ${ }^{a}$ Yun Chen, ${ }^{b}$ Gerald W. Dorn II, ${ }^{b}$ and Lihong V. Wang ${ }^{a, \star}$ \\ awashington University in St. Louis, Department of Biomedical Engineering, Optical Imaging Laboratory, One Brookings Drive, \\ St. Louis, Missouri 63130, United States \\ bWashington University in St. Louis, Department of Internal Medicine, Center for Pharmacogenomics, 660 S. Euclid, St. Louis, \\ Missouri 63110, United States
}

\begin{abstract}
Super-resolution microscopy techniques_capable of overcoming the diffraction limit of light-have opened new opportunities to explore subcellular structures and dynamics not resolvable in conventional far-field microscopy. However, relying on staining with exogenous fluorescent markers, these techniques can sometimes introduce undesired artifacts to the image, mainly due to large tagging agent sizes and insufficient or variable labeling densities. By contrast, the use of endogenous pigments allows imaging of the intrinsic structures of biological samples with unaltered molecular constituents. Here, we report label-free photoacoustic (PA) nanoscopy, which is exquisitely sensitive to optical absorption, with an $88 \mathrm{~nm}$ resolution. At each scanning position, multiple PA signals are successively excited with increasing laser pulse energy. Because of optical saturation or nonlinear thermal expansion, the PA amplitude depends on the nonlinear incident optical fluence. The high-order dependence, quantified by polynomial fitting, provides super-resolution imaging with optical sectioning. PA nanoscopy is capable of super-resolution imaging of either fluorescent or nonfluorescent molecules. () The Authors. Published by SPIE under a Creative Commons Attribution 3.0 Unported License. Distribution or reproduction of this work in whole or in part requires full attribution of the original publication, including its DOI. [DOI: 10.1117/1.JBO.19.8.086006]
\end{abstract}

Keywords: photoacoustics; super-resolution; microscopy; nanoscopy; mitochondria; label-free.

Paper 140197R received Apr. 28, 2014; revised manuscript received Jul. 3, 2014; accepted for publication Jul. 11, 2014; published online Aug. 7, 2014.

\section{Introduction}

Optical microscopy allows three-dimensional (3-D) imaging of living cells, tissues, and organisms. However, due to optical diffraction, conventional far-field microscopy is limited to imaging features no smaller than 200 to $300 \mathrm{~nm}$ laterally and 500 to $700 \mathrm{~nm}$ axially. ${ }^{1}$ Over the last decade, several super-resolution microscopy techniques have been developed to overcome the diffraction limit. ${ }^{2-7}$ Some of the techniques utilize nonlinear effects to narrow the point spread function (PSF) ${ }^{3-5}$ others use stochastic activation and averaging to localize individual fluorescent molecules. ${ }^{6,7}$ These fluorescence-based nanoscopy methods have improved both lateral and axial resolution by an order of magnitude over conventional microscopy. However, fluorescence tagging requires additional staining steps and, at times, can introduce image artifacts. ${ }^{1}$ Therefore, label-free imaging has been a major research impetus. Recently, autofluorescence-based nanoscopy of fluorophores, ${ }^{8}$ phase-based nanoscopy of specimens featuring precipitous refractive index changes, ${ }^{9}$ and photothermal microscopy of gold nanoparticles have been demonstrated. ${ }^{10}$ However, label-free nanoscopy of biological structures with chromophores having no or low fluorescence quantum yields-such as hemoglobin, cytochromes, melanin, DNA, and RNA-remains elusive.

Photoacoustic microscopy (PAM) is an effective in vivo functional and molecular imaging tool based on optical absorption contrast. ${ }^{11,12}$ In the PA phenomenon, light is absorbed by molecules and converted to heat. The subsequent thermoelastic expansion generates an acoustic wave. ${ }^{11,13}$ In conventional PAM, the PA signal is generated following excitation by a single

*Address all correspondence to: Lihong V. Wang, E-mail: Ihwang@ wustl.edu laser pulse per pixel, and the amplitude of the PA signal is assumed to be proportional to the excitation pulse energy. As light intensity increases, however, mechanisms such as thermal nonlinearity, ${ }^{14}$ optical saturation, ${ }^{15}$ or multiphoton absorption ${ }^{16}$ engender a significant nonlinear dependence on the excitation pulse energy. PA nonlinearity has recently been used in several applications, such as quantifying picosecond absorption relaxation times with a nanosecond laser ${ }^{15}$ and measuring oxygen saturation in vivo. ${ }^{17}$ Previously, we combined photoacoustics with the intensity-dependent photobleaching effect to demonstrate subdiffraction imaging of red blood cells and melanoma. ${ }^{18}$ Based on photobleaching of the absorbing molecules, this technique is destructive but tolerable. ${ }^{19}$ Here, we use nondestructive, nonlinear photoacoustics to achieve PA nanoscopy of biological structures having undetectable fluorescence, which adds a new contrast mechanism to the realm of nanoscopy.

\section{Methods and Materials}

\subsection{Principles of PA Nanoscopy}

Nonlinear PA effects arise from two major sources: nonlinear thermal expansion and optical absorption saturation. First, the thermal expansion coefficient $\beta(T)$ depends on the temperature rise $T$ above the equilibrium temperature. ${ }^{20,21}$ In thermal confinement, $T$ is proportional to the optical energy deposition. When $T$ is small, $\beta(T) \approx \beta_{1}+\beta_{2} T$, where $\beta_{1}$ and $\beta_{2}$ are the first two coefficients in the Taylor expansion around the equilibrium temperature. ${ }^{20}$ In water and soft tissues at room temperature, ${ }^{21} \beta_{2} \approx 0.04 \beta_{1}$, and, therefore, a temperature rise of $3 \mathrm{~K}$ can change the thermal expansion coefficient by $>10 \%$.

In our system, the laser pulse duration $(\sim 1.6 \mathrm{~ns})$ is much shorter than the transducer response time (several tens of 
nanoseconds), and, therefore, PA excitation is in stress confinement within the acoustically defined resolution. In stress confinement, the initial pressure rise, $p_{0}$, centered at $\vec{r}_{0}$ laterally and at $z_{0}$ axially, within the acoustic voxel, $V$, is given by

$p_{0}\left(\vec{r}_{0}, z_{0}\right)=\frac{1}{V_{0}} \int_{V} p\left(\vec{r}-\vec{r}_{0}, z-z_{0}\right) \cdot \mathrm{d} \vec{r} \mathrm{~d} z$

where $V_{0}$ is the volume of the acoustic voxel, and $p\left(\vec{r}-\vec{r}_{0}\right.$, $\left.z-z_{0}\right)$ is the laser-induced pressure at each point within the acoustic voxel given by ${ }^{20}$

$$
\begin{aligned}
p\left(\vec{r}-\vec{r}_{0}, z-z_{0}\right)= & \frac{1}{\kappa}\left\{\beta_{1} T\left(\vec{r}-\vec{r}_{0}, z-z_{0}\right)\right. \\
& \left.+\frac{1}{2} \beta_{2}\left[T\left(\vec{r}-\vec{r}_{0}, z-z_{0}\right)\right]^{2}\right\} .
\end{aligned}
$$

Here, $\kappa$ is the isothermal compressibility.

The diameter of the optical excitation area $(226 \mathrm{~nm})$ is large compared to the thermal diffusion length $(15 \mathrm{~nm}$ in water for a 1.6-ns laser pulse), and, therefore, the PA excitation is also in thermal confinement within the optically defined lateral resolution. In thermal confinement, the local temperature rise, $T\left(\vec{r}-\vec{r}_{0}, z-z_{0}\right)$, is given by ${ }^{22}$

$$
T\left(\vec{r}-\vec{r}_{0}, z-z_{0}\right)=\eta_{\mathrm{th}} A_{e}\left(\vec{r}-\vec{r}_{0}, z-z_{0}\right) /\left(\rho C_{p}\right),
$$

where $\eta_{\text {th }}$ is the percentage of deposited optical energy that is converted into heat, $A_{e}$ denotes the optical energy deposition $\left(\mathrm{J} / \mathrm{m}^{3}\right), \rho$ denotes the mass density, and $C_{p}$ denotes the specific heat capacity at constant pressure.

The second source of nonlinear PA effects is saturation of optical absorption. The energy deposition due to single photon absorption is

$$
\begin{aligned}
A_{e}\left(\vec{r}-\vec{r}_{0}, z-z_{0}\right)= & \int_{-\infty}^{\infty} \mu_{a}\left(\vec{r}-\vec{r}_{0}, z-z_{0}, t\right) \\
& \times I\left(\vec{r}-\vec{r}_{0}, z-z_{0}, t\right) \mathrm{dt},
\end{aligned}
$$

where $\mu_{a}\left(\vec{r}-\vec{r}_{0}, z-z_{0}, t\right)$ is the optical absorption coefficient $\left(\mathrm{m}^{-1}\right)$ at time $t$, and $I\left(\vec{r}-\vec{r}_{0}, z-z_{0}, t\right)$ is the optical intensity $\left(\mathrm{W} / \mathrm{m}^{2}\right)$. The optical absorption coefficient commonly saturates with increasing intensity in the form ${ }^{23}$

$\mu_{a}\left(\vec{r}-\vec{r}_{0}, z-z_{0}, t\right)=\frac{\mu_{a 0}(\vec{r}, z)}{1+\hat{I}\left(\vec{r}-\vec{r}_{0}, z-z_{0}, t\right)}$,

where $\mu_{a 0}(\vec{r}, z)$ is the unsaturated optical absorption coefficient, $\hat{I}\left(\vec{r}-\vec{r}_{0}, z-z_{0}, t\right)=I\left(\vec{r}-\vec{r}_{0}, z-z_{0}, t\right) / I_{\text {sat }}$, and $I_{\text {sat }}$ is the saturation intensity.

The optical intensity is presumed to have the following general form:

$$
I\left(\vec{r}-\vec{r}_{0}, z-z_{0}, t\right)=E_{p} \hat{f}_{s}\left(\vec{r}-\vec{r}_{0}, z-z_{0}\right) \hat{f}_{t}(t) .
$$

Here, $E_{p}$ is the pulse energy, which was varied for each voxel in PA nanoscopy. $\hat{f}_{s}\left(\vec{r}-\vec{r}_{0}, z-z_{0}\right)$ is the normalized spatial function. For a Gaussian beam,

$$
\hat{f}_{s}\left(\vec{r}-\vec{r}_{0}, z-z_{0}\right)=\frac{1}{\pi\left[w\left(z-z_{0}\right)\right]^{2}} \exp \left\{-\frac{2\left(\vec{r}-\vec{r}_{0}\right)^{2}}{\left[w\left(z-z_{0}\right)\right]^{2}}\right\}
$$

where $w(z)$ is the beam width given by

$$
w\left(z-z_{0}\right)=w_{0} \sqrt{1+\left(\frac{z-z_{0}}{z_{R}}\right)^{2}} .
$$

Here, $w_{0}$ is the waist radius and $z_{R}=\pi w_{0}^{2} / \lambda$ is the Rayleigh length. $\hat{f}_{t}(t)$ is the normalized temporal function. For a Gaussian pulse,

$\hat{f}_{t}(t)=\frac{1}{\sqrt{2 \pi} \tau_{L}} \exp \left[-\frac{t^{2}}{2 \tau_{L}^{2}}\right]$

where $\tau_{L}$ is a parameter related to the full width at half maximum (FWHM) of the pulse $\left[\mathrm{FWHM}=2 \sqrt{2 \ln (2)} \tau_{L}\right]$, which was constant in our experiments.

Following Beer's law, in the presence of optical absorption/ scattering, the optical intensity also includes attenuation with depth $z$. However, when the thickness of the sample (e.g., 3 to $10 \mu \mathrm{m})$ is much smaller than the characteristic penetration depth (e.g., for hemoglobin in red blood cells, $1 / \mu_{a 0} \cong 44 \mu \mathrm{m}$ at $532 \mathrm{~nm}$, and the scattering effect is negligible), the Born approximation is valid, i.e., the Beer's law attenuation term is negligible.

Substitution of Eq. (5) into Eq. (4) yields

$$
\begin{aligned}
& A_{e}\left(\vec{r}-\vec{r}_{0}, z-z_{0}\right) \\
& \quad=\mu_{a 0}(\vec{r}, z) I_{\text {sat }} \int_{-\infty}^{\infty} \frac{\hat{I}\left(\vec{r}-\vec{r}_{0}, z-z_{0}, t\right)}{1+\hat{I}\left(\vec{r}-\vec{r}_{0}, z-z_{0}, t\right)} \mathrm{d} t .
\end{aligned}
$$

Because $\hat{I}$ was less than unity in our experiments, we can expand the integrand to the $n$ 'th order as follows:

$$
\begin{aligned}
A_{e}\left(\vec{r}-\vec{r}_{0}, z-z_{0}\right) \approx & \mu_{a 0}(\vec{r}, z) I_{\mathrm{sat}} \int_{-\infty}^{\infty}\left[\hat{I}-\hat{I}^{2}+\hat{I}^{3} \ldots\right. \\
& \left.+(-1)^{n-1} \cdot \hat{I}^{n}\right] \mathrm{dt} .
\end{aligned}
$$

For a Gaussian pulse, Eq. (11) becomes

$$
\begin{aligned}
A_{e}\left(\vec{r}-\vec{r}_{0}, z-z_{0}\right) \approx & \mu_{a 0}(\vec{r}, z) F_{\text {sat }}\left[\sqrt{\frac{1}{1}} \hat{F}-\sqrt{\frac{1}{2}} \hat{F}^{2}\right. \\
& \left.+\sqrt{\frac{1}{3}} \hat{F}^{3} \ldots+(-1)^{n-1} \sqrt{\frac{1}{n}} \hat{F}^{n}\right],
\end{aligned}
$$

where $F_{\text {sat }}=\sqrt{2 \pi} \tau_{L} \mathrm{I}_{\text {sat }}$, and $\hat{F}$ is the fluence normalized by $F_{\text {sat }}$, $\hat{F}=\left(E_{p} / F_{\text {sat }}\right) \hat{f}_{s}\left(\vec{r}-\vec{r}_{0}, z-z_{0}\right)$. Substitution of Eqs. (12), (3), and (2) into Eq. (1) yields

$p_{0}\left(\vec{r}_{0}, z_{0}, E_{p}\right)=\sum_{n=1}^{\infty} c_{n}\left(\vec{r}_{0}, z_{0}\right) \cdot\left(E_{p}\right)^{n}$ 


$$
\begin{aligned}
c_{n}\left(\vec{r}_{0}, z_{0}\right)= & \frac{1}{V_{0}} \int_{V}\left\{(-1)^{n-1} \Gamma_{1}(n) \eta_{\mathrm{th}} F_{\mathrm{sat}} \mu_{n}^{\prime}(\vec{r}, z)\right. \\
& \left.\times\left[\frac{\hat{f}_{s}\left(\vec{r}-\vec{r}_{0}, z-z_{0}\right)}{F_{\text {sat }}}\right]\right\} \mathrm{d} \vec{r} \mathrm{~d} z .
\end{aligned}
$$

Here,

$$
\begin{aligned}
\mu_{n}^{\prime}(\vec{r}, z) & =\mu_{a 0}(\vec{r}, z)\left[1-\frac{\gamma_{2}(n) \eta_{\mathrm{th}} F_{\mathrm{sat}} \mu_{a 0}(\vec{r}, z)}{\Gamma_{1}(n)}\right], \\
\Gamma_{1}(n) & =\beta_{1} /\left(\kappa \rho C_{p} \sqrt{n}\right) \quad n \geq 1, \\
\gamma_{2}(n) & = \begin{cases}0 & n=1 \\
\beta_{2} /\left[2 \kappa\left(\rho C_{p}\right)^{2}\right] \cdot\left[\sum_{m=1}^{n-1} \sqrt{\frac{1}{m(n-m)}}\right] & n>1\end{cases}
\end{aligned}
$$

The image constructed from each coefficient, $c_{n}\left(\vec{r}_{0}, z_{0}\right)$, in Eq. (14) is a convolution between $\left[\hat{f}_{s}\left(\vec{r}-\vec{r}_{0}, z-z_{0}\right)\right]^{n}$ and a modified absorption distribution term, $\mu_{n}^{\prime}(\vec{r}, z)$, which can be considered a compound contrast mechanism for PA nanoscopy. In the linear regime, the first coefficient, $c_{1}\left(\vec{r}_{0}, z_{0}\right)$, is a convolution between $\hat{f}_{s}\left(\vec{r}-\vec{r}_{0}, z-z_{0}\right)$ and $\mu_{a 0}(\vec{r}, z)$. The corresponding lateral PSF for $c_{1}\left(\vec{r}_{0}, z_{o}\right)$ has an optical diffraction-limited width of $0.51 \cdot \lambda / \mathrm{NA}$, where $\lambda$ is the optical wavelength and NA is the numerical aperture of the optical objective lens. Higherorder coefficients contain higher powers of fluence distribution, thus effectively narrowing the lateral PSF and enabling imaging with spatial resolution finer than the diffraction limit. The interplay between $\Gamma_{1}(n)$ and $\gamma_{2}(n)$ improves the resolution of the high-order coefficients $c_{n}$ in Eq. (14). When either $\Gamma_{1}(n)$ or $\gamma_{2}(n)$ dominates, $c_{n}$ follows $\hat{f}_{s}^{n}$, improving the lateral resolution by a factor of $\sqrt{n}$ over that of $c_{1}$ (i.e., linear PAM). However, when $\Gamma_{1}(n) \cong 0.5 \cdot \gamma_{2}(n) \eta_{\mathrm{th}} F_{\mathrm{sat}} \mu_{\mathrm{a} 0}^{\max }$, the resolution is further improved.

To extract the coefficients, $c_{n}\left(\vec{r}_{0}, z_{0}\right)$, of the PA signal up to the $N$ 'th order, a train of $M \geq N$ pulses with different pulse energies incident at the same position can be used. A linear least-squares estimation gives

$$
\left[\begin{array}{c}
c_{1} \\
\vdots \\
c_{N}
\end{array}\right]=\left(V^{T} V\right)^{-1} V^{T} \times\left[\begin{array}{c}
p_{1} \\
\vdots \\
p_{M}
\end{array}\right] .
$$

Here, $p_{j}$ is the PA signal generated by the $j$ 'th pulse $(j=1 \ldots M)$, centered at position $\vec{r}_{0}$ laterally and at $z_{0}$ axially, and $V$ is a Vandermonde matrix whose elements are powers of the pulse energies.

$$
V=\left[\begin{array}{cccc}
E_{p_{1}} & E_{p_{1}}^{2} & \cdots & E_{p_{1}}^{N} \\
E_{p_{2}} & E_{p_{2}}^{2} & \cdots & E_{p_{2}}^{N} \\
\vdots & \vdots & \ddots & \vdots \\
E_{p_{M}} & E_{p_{M}}^{2} & \cdots & E_{p_{M}}^{N}
\end{array}\right]
$$

\subsection{Optical Sectioning of PA Nanoscopy}

To evaluate the optical sectioning capability of PA nanoscopy, we consider a slab-shaped compound absorption coefficient, $\mu_{n}^{\prime}(\vec{r}, z)$, with an infinitesimally thin thickness, i.e., $\mu_{n}^{\prime}(\vec{r}, z) \propto$ $\delta\left(z-z^{\prime}\right)$. By taking the integration in Eq. (14) for this sample, the coefficients can be presented as a function of $z_{0}$.

$c_{n}\left(z_{0}\right) \propto \frac{1}{\left[w\left(z_{0}-z^{\prime}\right)\right]^{2(n-1)}}$.

Normalization to the peak value at $z_{0}=z^{\prime}$, the slab's true location, gives

$$
\frac{c_{n}\left(z_{0}\right)}{c_{n}\left(z_{0}=z^{\prime}\right)}=\left[1+\left(\frac{z_{0}-z^{\prime}}{z_{R}}\right)^{2}\right]^{-(n-1)} .
$$

In the linear regime, $c_{1}\left(z_{0}\right)$ is constant with $z_{0}$, and, therefore, there is no optical sectioning. However, for higher orders, $c_{n}\left(z_{0}\right)$ narrows around $z_{0}=z^{\prime}$. The expected optical sectioning capability of the $n$ 'th coefficient, $\Delta z_{n}$, can be quantified as the FWHM of $c_{n}\left(z_{0}\right)$.

$\Delta z_{n}=2 z_{R} \cdot \sqrt{\sqrt[n-1]{2}-1}$

For example, the expected optical sectioning capabilities are $2.0 z_{R}, 1.3 z_{R}$, and $1.0 z_{R}$ for $c_{2}, c_{3}$, and $c_{4}$, respectively.

\subsection{Experimental Setup of PA Nanoscopy}

In the scheme depicted in Fig. 1(a), an Nd:YVO4 laser (Spot 10-200-532, Elforlight, Daventry, United Kingdom) generates a train of 1.6-ns pulses at $532 \mathrm{~nm}$ with a $2.35 \mathrm{kHz}$ pulse repetition rate. To allow per-pulse energy variation, the laser beam is passed through an electro-optic modulator (350-50, Conoptics, Danbury, Connecticut), which is controlled by LabVIEW (National Instruments, Austin, Texas) and synchronized with the laser trigger. The beam is then spatially filtered, partially sampled by a photodiode (S1226-18BK, Hamamatsu, Bridgewater, New Jersey), and focused onto the sample using an infinity corrected objective (NA 1.20, 60×, UPlanSApo, Olympus, Center Valley, Pennsylvania). The focus is precisely adjusted using a piezoelectric actuator (PAS080, Thorlabs, Newton, New Jersey). The laser pulse energy at the target ranges from 0.5 to $5 \mathrm{~nJ}$ for imaging melanoma cells, 20 to $100 \mathrm{~nJ}$ for imaging mitochondria, and 0.01 to $0.25 \mathrm{~nJ}$ for imaging gold nanoparticles. A train of four pulses with varied pulse energies is fired for each pixel. The photodiode is used to accurately measure the energy of each pulse for pulse-to-pulse signal calibration. The PA signal is detected by an ultrasonic transducer $(40 \mathrm{MHz}$ central frequency and NA 0.5). After amplification, the PA signal is digitized at a sampling rate of $500 \mathrm{MHz}$ using a data acquisition card (ATS9350, Alazartech, Pointe-Claire, Quebec, Canada). A piezoelectric scanning stage (NPXY400A, nPoint, Middleton, Wisconsin) raster scans the objective lens and the ultrasonic transducer with a step size of $25 \mathrm{~nm}$ in the $x y$-plane. Coefficients $c_{n}\left(\vec{r}_{0}, z_{0}\right)$ are extracted by fitting the PA dependence on pulse energy with a polynomial. Both conventional PAM and PA nanoscopy images were processed the same way by passing the raw data (for conventional PAM) or the highorder coefficient images (for PA nanoscopy) through a $3 \times 3$ low-pass Wiener filter to remove high-frequency noise. In the current embodiment of PA nanoscopy, the acquisition time for an image with $256 \times 256$ pixels is $\sim 112 \mathrm{~s}$. 
(a)

Input pulse train

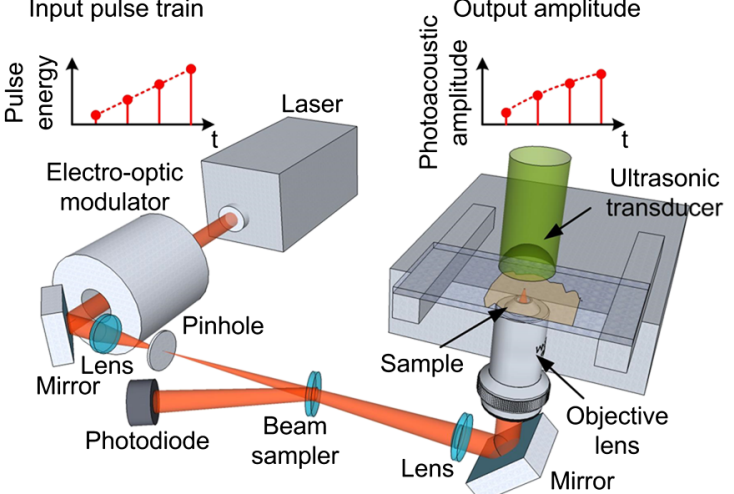

(b)

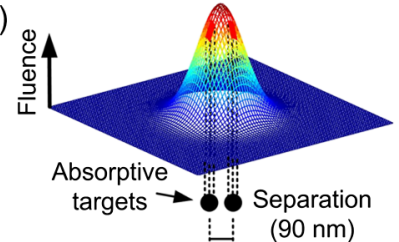

(d)

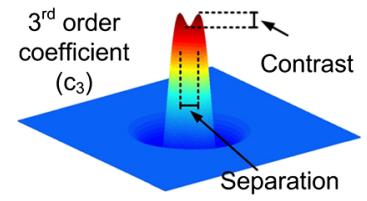

(c) Linear

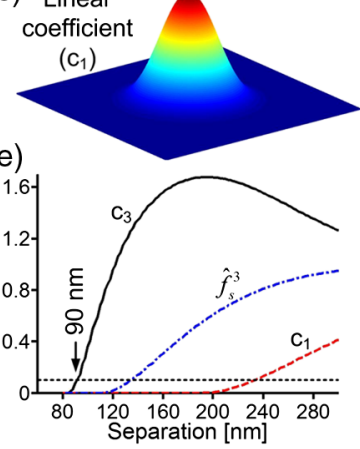

Fig. 1 Principle of photoacoustic (PA) nanoscopy. (a) Schematic of the PA nanoscope. At each scanning position, a train of pulses with increasing energy successively excites PA signals. Due to optical saturation or nonlinear thermal expansion, the PA amplitude increases nonlinearly with the increasing incident energy. (b) Fluence distribution of a Gaussian illumination beam of $226 \mathrm{~nm}$ full width at half maximum, which is scanned over two absorbers $90 \mathrm{~nm}$ apart. (c) Image from the normalized linear PA coefficient, $c_{1}$, of the two absorbers. Proportional to $\mu_{a 0} \otimes f_{s}, c_{1}$ is diffraction limited. (d) Image from normalized $c_{3}$, which resolves the two particles. (e) Contrast as a function of the separation between the two particles for $c_{1}, \hat{f}_{s}^{3}$, and $c_{3}$ when $\Gamma_{1}(n=3)=$ $0.5 \cdot \gamma_{2}(n=3) \eta_{\mathrm{th}} F_{\mathrm{sat}} \mu_{20}^{\max }$. The spatial resolutions as defined by the particle separations at $10 \%$ contrast (horizontal dashed line) are $\sim 234, \sim 135$, and $\sim 90 \mathrm{~nm}$, respectively.

\subsection{Culture of Melanoma Cells and Fibroblasts}

Genetically modified mouse embryonic fibroblasts (MEFs), NIH-3T3 cells, and B16 melanoma cells were obtained from American Type Culture Collection (Manassas, Virginia) and maintained in Dulbecco's modified Eagle medium (Invitrogen, Carlsbad, California) supplemented with $10 \%$ fetal bovine serum (Invitrogen) and $1 \%$ penicillin-streptomycin (P/S, Invitrogen) at $37^{\circ} \mathrm{C}$ and $5 \% \mathrm{CO}_{2}$. The cells were seeded onto a cover glass at a density of $\sim 1 \times 10^{4}$ cells $/ \mathrm{cm}^{2}$ and grown overnight. The cells were then fixed in $4 \%$ formaldehyde and washed with phosphate buffered saline three times. For confocal imaging, MEF cells were stained with $200 \mathrm{nM}$ Mitotracker-red for $10 \mathrm{~min}$ before fixation.

\subsection{Cover Glass Photolithography}

Glass coverslips $(24 \mathrm{~mm} \times 40 \mathrm{~mm}$, Corning, Lowell, Massachusetts) were deposited with micron-sized chromium patterns that served as landmarks for imaging gold nanoparticles. First, each glass coverslip was cleaned with acetone and washed thoroughly with distilled water. Second, a $1.5-\mu \mathrm{m}$-thick layer of photoresist (AZ5314) was spin-coated onto the substrate, followed by prebaking at $80^{\circ} \mathrm{C}$ for $1 \mathrm{~min}$. Third, a negative photomask was placed upon the photoresist and both were exposed to $0.08 \mathrm{~J} / \mathrm{cm}^{2}$ ultraviolet light for $10 \mathrm{~s}$. After the photomask was removed, the photoresist was reverse-baked at $90^{\circ} \mathrm{C}$ for $2 \mathrm{~min}$ and re-exposed to $0.08 \mathrm{~J} / \mathrm{cm}^{2}$ ultraviolet light for $30 \mathrm{~s}$. The coverslip was then developed for $30 \mathrm{~s}$ by a metal-ion-free photoresist developer (AZ327). Fourth, using a thermal evaporator, a 50-nm-thick chromium layer was deposited on the photoresist patterns. Finally, the photoresist, together with the metal on top of it, was removed in acetone, leaving chromium patterns on the glass substrate.

\subsection{Fabrication and Characterization of a Colloidal Gold Monolayer on Glass}

Glass coverslips ( $24 \mathrm{~mm} \times 40 \mathrm{~mm}$, Corning) with micron-sized chromium patterns were used as substrates for assembly of the colloidal gold monolayer. The glass substrates were cleaned by sonication for $5 \mathrm{~min}$ in hot RBS 35 detergent (Thermo Scientific, Waltham, Massachusetts) and washed extensively with distilled water. The cleaned glass substrates were subjected to plasma oxidation for $30 \mathrm{~min}$ and then immersed in a $10 \%$ $(\mathrm{v} / \mathrm{v})$ solution of (3-Aminopropyl)triethoxysilane (SigmaAldrich, Saint Louis, Missouri) in anhydrous ethanol for $20 \mathrm{~min}$, rinsed five times in anhydrous ethanol with sonication for $3 \mathrm{~min}$ each time, and dried at $120^{\circ} \mathrm{C}$ for $3 \mathrm{~h}$. To attach the gold nanoparticles, $\sim 250 \mu \mathrm{l}$ of colloidal gold solution $\left(\sim 2 \times 10^{8}\right.$ particles $\left./ \mathrm{ml}\right)$ was left overnight on one side of the silanized glass coverslip in a $45^{\circ} \mathrm{C}$ oven. The glass coverslips were then washed in distilled water on a shaker for $3 \mathrm{~h}$ and dried in a $60^{\circ} \mathrm{C}$ oven for $1 \mathrm{~h}$. The immobilized colloids were imaged by atomic force microscopy (AFM) in the tapping mode in air, using standard silicon cantilevers (Nano World, Neuchâtel, Switzerland) on a Veeco Nanoman scanning probe microscope (Veeco Instruments, Plainview, New York).

\section{Results}

\subsection{Characteristics of PA Nanoscopy}

To illustrate the concept of PA nanoscopy using our actual experimental conditions (e.g., a 1.2 NA objective), we simulated $c_{1}$ and $c_{3}$ by assuming $\Gamma_{1}(n=3)=0.5 \cdot \gamma_{2}(n=3) \eta_{\text {th }} F_{\text {sat }} \mu_{a 0}^{\max }$. The lateral PSF for $c_{1}$ has an optical diffraction-limited width of $0.51 \cdot \lambda / \mathrm{NA}$, where $\lambda$ is the optical wavelength $(532 \mathrm{~nm}$ in our setup) and NA is the numerical aperture of the optical objective lens. Hence, we considered a Gaussian illumination beam of $226 \mathrm{~nm}$ FWHM and two absorbers with absorption coefficients following Gaussian distributions (each with a $5 \mathrm{~nm}$ FWHM diameter) located $90 \mathrm{~nm}$ apart [Fig. 1(b)]. While the image with diffraction-limited resolution from the linear PA coefficient, $c_{1}$, exhibited a single blurred peak [Fig. 1(c)], the image with super-resolution from $c_{3}$ [Fig. 1(d)] clearly resolved the two particles. Figure 1(e) shows the contrast (i.e., the depth of the dip divided by the peak signal) — as a function of the distance between the two particles-from $c_{1}$ for linear PAM, from $\hat{f}_{s}^{3}$ (i.e., $\left.c_{3}\right)$ for PA nanoscopy when $\Gamma_{1}(n=3)$ or $\gamma_{2}(n=3)$ dominates, and from $c_{3}$ when $\Gamma_{1}(n=3)=0.5 \cdot \gamma_{2}(n=3)$ $\eta_{\mathrm{th}} F_{\mathrm{sat}} \mu_{\mathrm{a} 0}^{\max }$. 


\subsection{Resolution Validation Using Gold Nanoparticles of $100 \mathrm{~nm}$ Diameter}

To verify the ability of PA nanoscopy to resolve nanoscopic features, we imaged 100-nm-diameter gold nanoparticles fixed on a glass coverslip. The particles were first imaged using AFM with $25 \mathrm{~nm}$ resolution and subsequently PA nanoscopy. A landmark pattern was deposited on the coverslip to ensure that the same area was imaged with both systems. Two pairs of adjacent gold nanoparticles were selected from the AFM images to validate PA images [Figs. 2(a) and 2(b), left column]. While conventional PAM [Figs. 2(a) and 2(b), middle column] cannot resolve the adjacent nanoparticles, PA nanoscopy images constructed from $c_{3}$ [Figs. 2(a) and 2(b), right column] clearly resolve them with center-to-center spacings of 200 and $110 \mathrm{~nm}$ and contrastto-noise ratios of 8 and 6, respectively [Figs. 2(c) and 2(d)]. When the illumination beam's center is adjacent to the gold nanoparticles [Fig. 2(e)], the PA signal increases linearly with fluence, yielding a zero $c_{3}$ at this point. When the illumination beam's center coincides with the center of a gold nanoparticle [Fig. 2(f)], the PA signal exhibits significant nonlinear effects. Naturally, the nonlinear effects weaken when the illumination's beam center is between the gold nanoparticles [Fig. 2(g), Video 1].

The saturation intensity of metal, such as gold or chromium, is much larger than that of organic molecules. In gold nanoparticles, therefore, thermal nonlinearity dominates ${ }^{14}$ and enables subdiffraction resolution even in conventional PAM, i.e., a single pulse can generate a significant nonlinear PA (or photothermal) component and the PSF may appear narrower than expected. ${ }^{10,24,25}$ However, the resolution of such a conventional PAM image may vary for different pulse energies. PA nanoscopy separates the nonlinear components from the linear components to enhance the spatial resolution. Here, a conventional PA image [Figs. 2(b), middle column, and 2(d)] taken at the highest pulse energy vaguely shows the gold nanoparticle

(a)
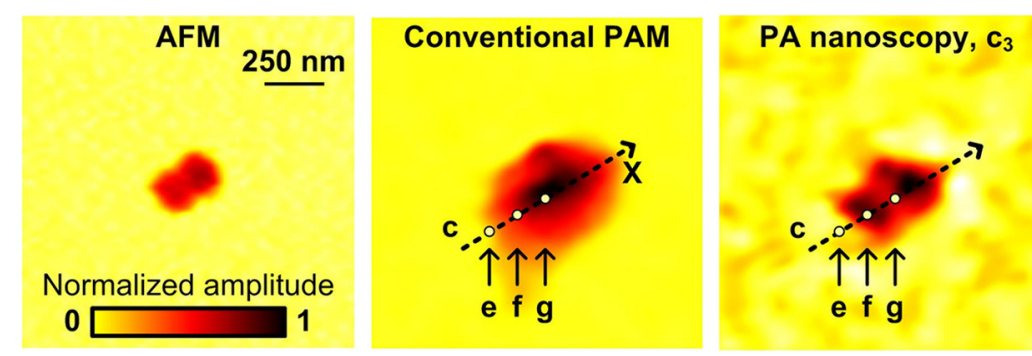

(b)
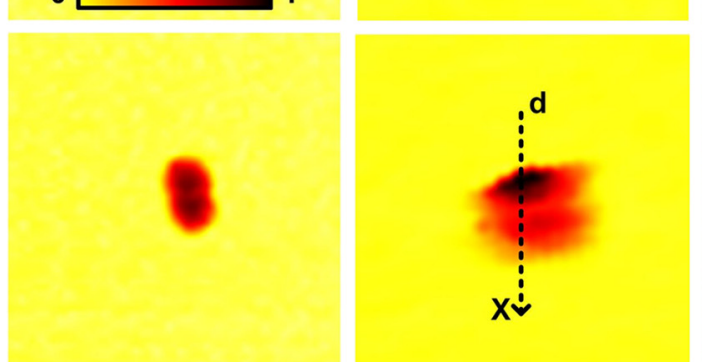

(c)

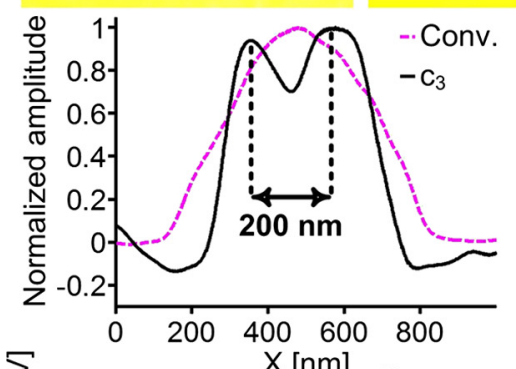

(e)
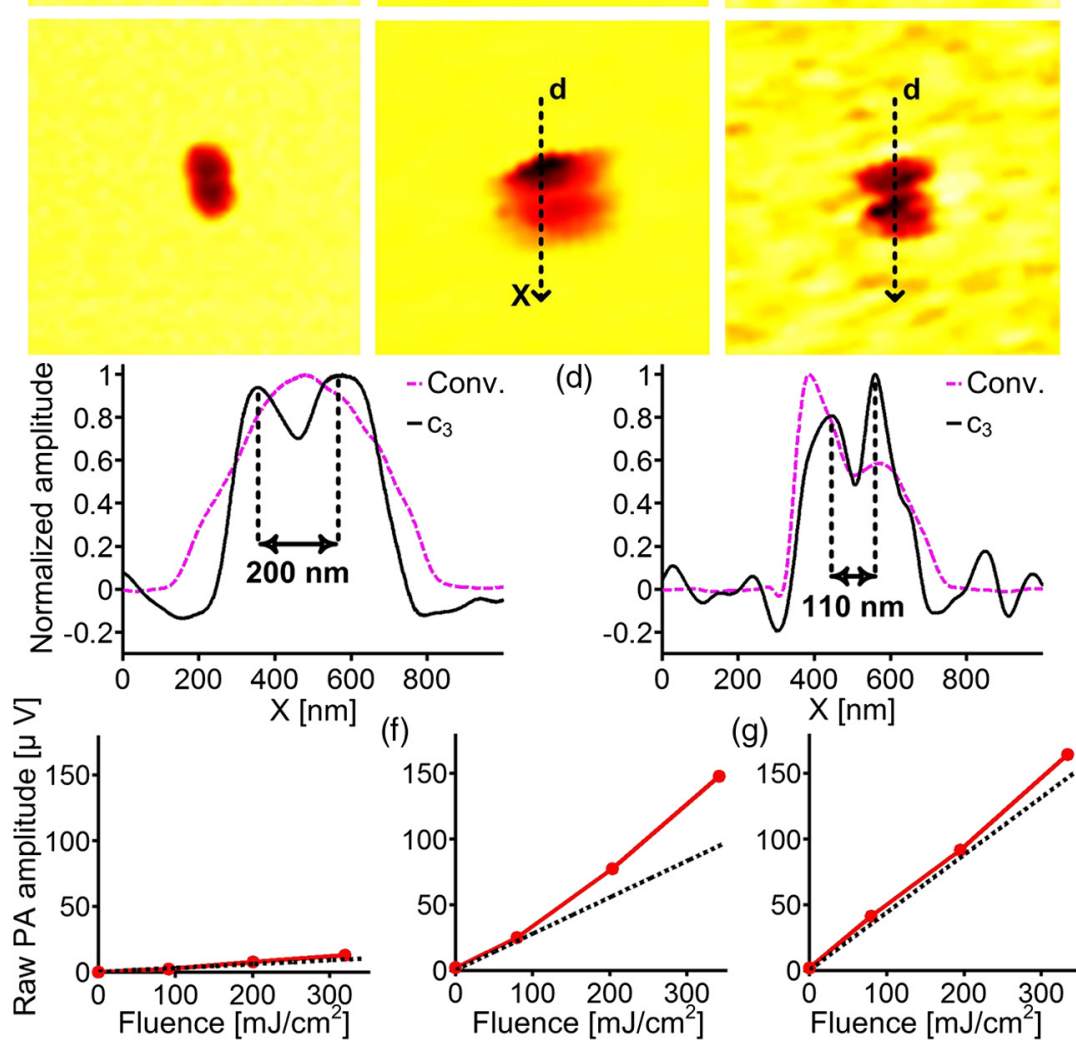

(g)

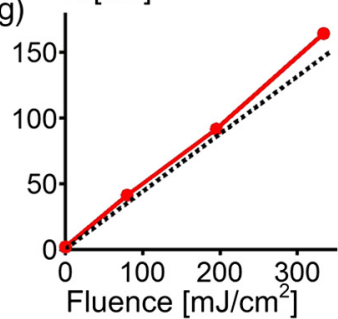

Fig. 2 Imaging gold nanoparticles of $100 \mathrm{~nm}$ diameter. (a) and (b) Images acquired with atomic force microscopy (left column), conventional PA microscopy (PAM) (middle column), and third-order PA nanoscopy, $c_{3}$ (right column). (c) and (d) Normalized image amplitude along the dashed lines in (a) and (b). (e) to (g) Raw PA amplitude as a function of the incident pulse fluence when the center of the beam is (e) outside, ( $f$ ) at the center, and ( $g$ ) between the gold nanoparticles. Each black dashed line in (e) to $(g)$ is the tangent of the nonlinear curve at the origin. The slope of the black dashed line represents the conventional linear PA amplitude (Video 1, QuickTime, 0.3 Mb) [URL: http://dx.doi.org/10.1117/1.JBO.19.8 $.086006 .1]$. 
pair. In comparison, the pair is revealed clearly by PA nanoscopy [Figs. 2(b), right column, and 2(d)].

\subsection{PA Nanoscopy of Mitochondria in Fibroblasts and Melanosomes in Melanoma Cells}

Next, we demonstrated label-free PA nanoscopy of mitochondria in fibroblasts and melanosomes in melanoma cells. Many mitochondrial haemoproteins, such as cytochrome $c$, as well as melanin, the primary absorber in melanotic melanoma cells, have strong optical absorption but negligible fluorescence quantum yield. ${ }^{26}$ Cytochromes are endogenous pigments that are highly specific to the inner mitochondrial membrane and were successfully used in photothermal and PA imaging of mitochondria. ${ }^{27,28}$ Figures 3(a) and 3(b) present a typical tubularshaped mitochondrion in a fibroblast (NIH 3T3) imaged by conventional PAM and third-order $\left(c_{3}\right)$ PA nanoscopy. A similar structure is revealed by transmission electron microscopy in Fig. 3(c). The $c_{3}$ image reveals the shape of a single mitochondrion, with features that are $88 \pm 12 \mathrm{~nm}$ apart [Fig. 3(d) and Video 2], which matches our simulation [Fig. 1(e)]. To demonstrate PA nanoscopy's ability to resolve different morphometries of mitochondria, we imaged genetically modified MEFs in which the normal interconnected mitochondrial network is fragmented to various degrees through ablation of one or both Mitofusin (Mfn) genes. ${ }^{29}$ The mitochondria in wild-type MEF cells show global interconnectivity (Fig. 4), while in the

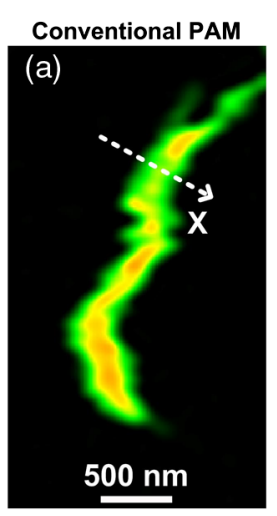

PA nanoscopy, $c_{3}$
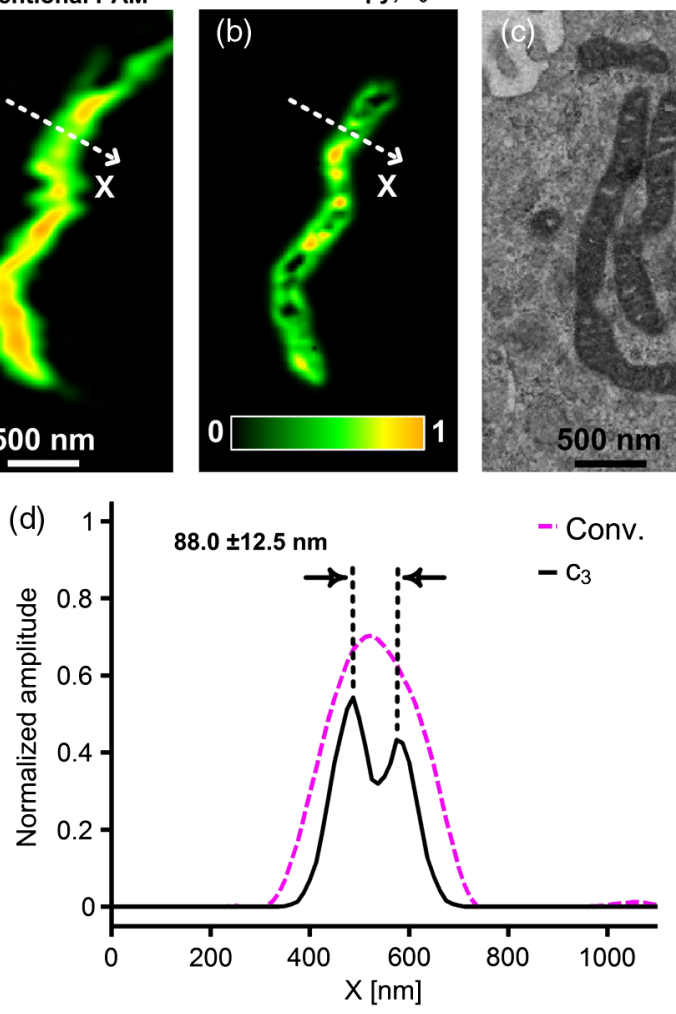

Fig. 3 Imaging mitochondria in NIH 3T3 fibroblasts. A typical tubular shaped mitochondrion imaged by (a) conventional PAM and (b) thirdorder $\left(c_{3}\right)$ PA nanoscopy. (c) A similar structure of mitochondria is revealed by transmission electron microscopy. (d) Normalized image amplitude along the dashed lines in (a) and (b) (Video 2, QuickTime, $0.1 \mathrm{Mb}$ ) [URL: http://dx.doi.org/10.1117/1.JBO.19.8 .086006.2].
Mfn knockout cells, the network is fragmented and individual organelles are readily resolved (Video 3). Melanoma cells were imaged with conventional PAM at different scales by varying the lateral step size (Fig. 5). Images of a cluster of melanosomes-small organelles containing melanin-were acquired using conventional PAM, second-order $\left(c_{2}\right)$, and third-order $\left(c_{3}\right)$ PA nanoscopy. In the $c_{3}$ image, unwanted background from outof-focus absorbers is rejected, revealing peaks with FWHMs as small as $80 \mathrm{~nm}$ and enabling a better estimation of the melanosomes' shapes and sizes (Video 4).

\subsection{Optically Sectioned PA Nanoscopy of Red Blood Cells and Mitochondria in Fibroblasts}

Finally, the nonlinear PA effects also provide optical sectioning, as in multiphoton microscopy, ${ }^{30}$ which improves the axial resolution dramatically. To demonstrate this improvement, we imaged red blood cells using a 1.2 NA objective (beam diameter, $226 \mathrm{~nm}$; depth of focus, $440 \mathrm{~nm}$ ) at eight different focal depths, with a step size of $500 \mathrm{~nm}$. By scanning with a lateral step size of $50 \mathrm{~nm}$ at each focal depth, the conventional PA image was acquired with low pulse energy, and the nonlinear PA image was constructed from $c_{2}$. Representative cross-sectional images of the red blood cells from conventional PAM and from PA nanoscopy are shown in Figs. 6(a) and 6(b), respectively (Videos 5 and 6). In the conventional PA image, out-of-plane objects are blurred but not rejected, yielding a signal-to-background ratio (SBR) of merely 2. PA nanoscopy, in contrast, detects only the features that reside within the depth of focus, rejecting background from out-of-focus absorbers (SBR $\cong 16$ ). Figures 6(c) and 6(d) show three-dimensionally rendered structural images of mitochondria acquired at eight focal depths, $300 \mathrm{~nm}$ apart, by conventional PAM and third-order $\left(c_{3}\right)$ PA nanoscopy. Because the thickness of the fibroblast $(\sim 4 \mu \mathrm{m})$ is greater than the depth of focus, the conventional PA image [Fig. 6(c)] contains unwanted background from out-of-focus absorbers. In comparison, owing to its intrinsic optical sectioning capability, PA nanoscopy [Fig. 6(d)] achieves a much higher SBR (Video 7).

\section{Discussion}

For nanosecond-pulsed laser light, heating is the dominant mechanism of potential damage in biological tissue. To cause damage, the instantaneous temperature rise must be $>150^{\circ} \mathrm{C}$, or the cumulative temperature rise must reach $43^{\circ} \mathrm{C}$ for a sustained period of time, e.g., $>60 \mathrm{~s} .{ }^{31,32}$ In PA nanoscopy of biological samples, the instantaneous local temperature rise may reach tens of degrees. For example, at $532 \mathrm{~nm}$, the absorption coefficient of cytochromes in mitochondria is $\sim 1 \mathrm{~cm}^{-1} .^{27}$ Hence, for a fluence of $60 \mathrm{~J} / \mathrm{cm}^{2}$, the local temperature rise is estimated to be $\sim 15 \mathrm{~K}$. However, despite repeated imaging (e.g., Figs. 5 and 7, Videos 1 and 5), no evidence of photodamage or alteration to the cellular appearance was found. To further reduce the local temperature rise, shorter laser pulses with lower energy can be used. Thus, the pulse intensity can still approach the saturation intensity level, while the incident fluence is reduced. For gold nanoparticles, the local temperature rise is inversely proportional to the particles' diameter. ${ }^{19}$ We found experimentally that particles $>100 \mathrm{~nm}$ in diameter could be imaged by PA nanoscopy without observable photobleaching.

In PA nanoscopy, the nonlinear PA signal depends on the nonlinear absorption coefficient. Nonlinear dependence of the signal amplitude on molecule concentration exists in other 


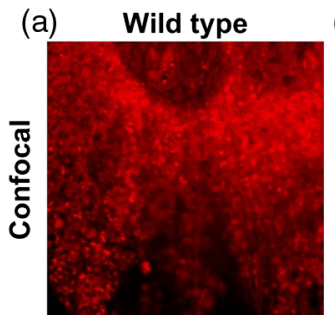

(e)

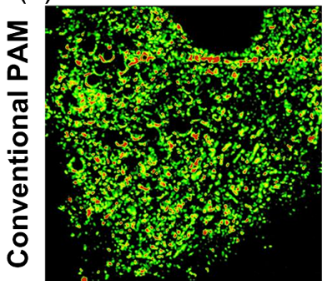

(i)
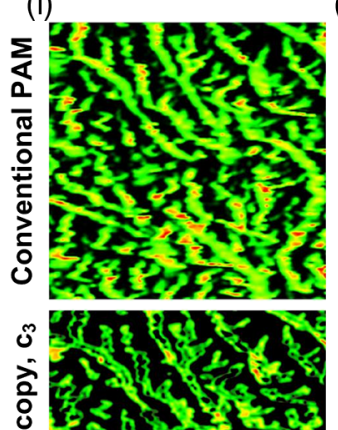

O

$\stackrel{5}{\frac{1}{5}}$

更

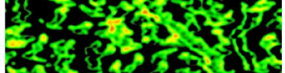

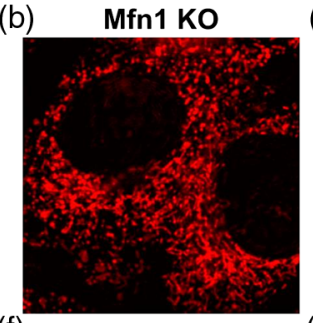

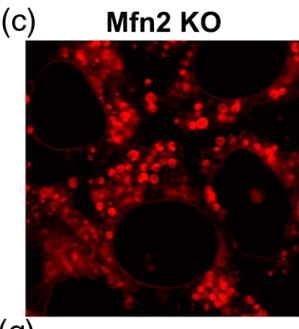

(g)

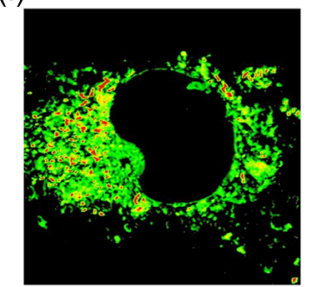

(1)
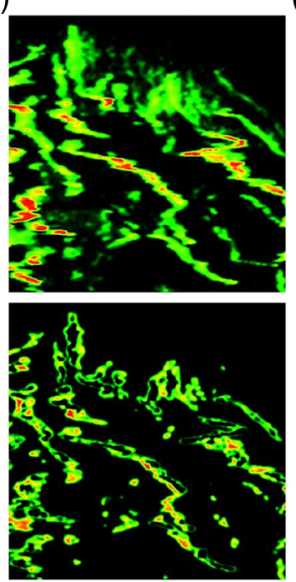
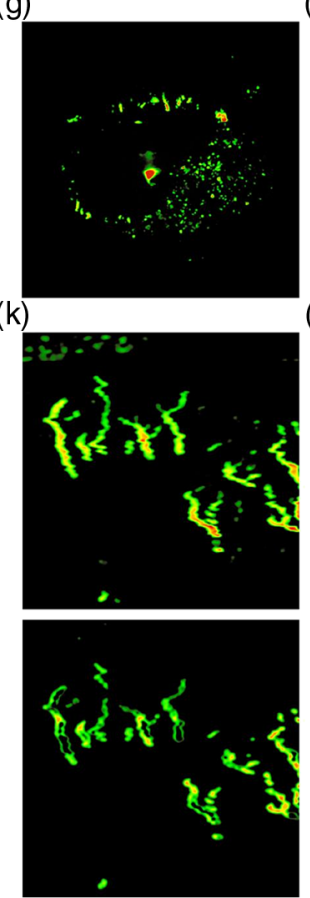

(d) Mfn1,Mfn2 DKo

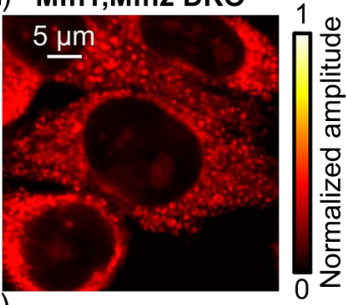

h)
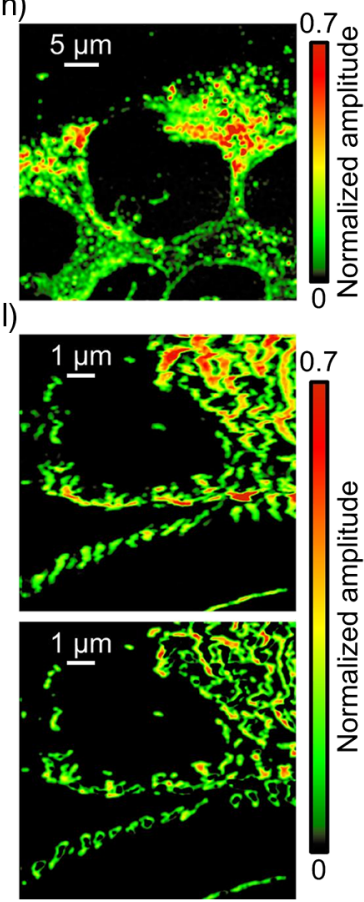

Fig. 4 Imaging mouse embryonic fibroblasts (MEFs). Confocal microscopy images of (a) a wild-type MEF, (b) MEFs in which the Mitofusin-1 gene was knocked out (Mfn1-KO), (c) MEFs in which the Mitofusin-2 gene was knocked out (Mfn2-KO), and (d) MEFs in which both Mfn1 and Mfn2 were knocked out (Mfn1,Mfn2DKO). Conventional PAM images of (e) a wild-type MEF, (f) Mfn1-KO, (g) Mfn2-KO, and (h) Mfn1,Mfn2DKO. Conventional PAM and third-order $\left(c_{3}\right)$ PA nanoscopy images of mitochondria in (i) a wild-type MEF, (j) Mfn1-KO, (k) Mfn2-KO, and (I) Mfn1,Mfn2-DKO. The mitochondria in wild-type MEF cells show global interconnectivity, while in the Mfn knockout cells, the network is fragmented and individual organelles are readily resolved (Video 3, QuickTime, 0.7 Mb) [URL: http://dx.doi.org/10.1117/1.JBO.19.8.086006.3].
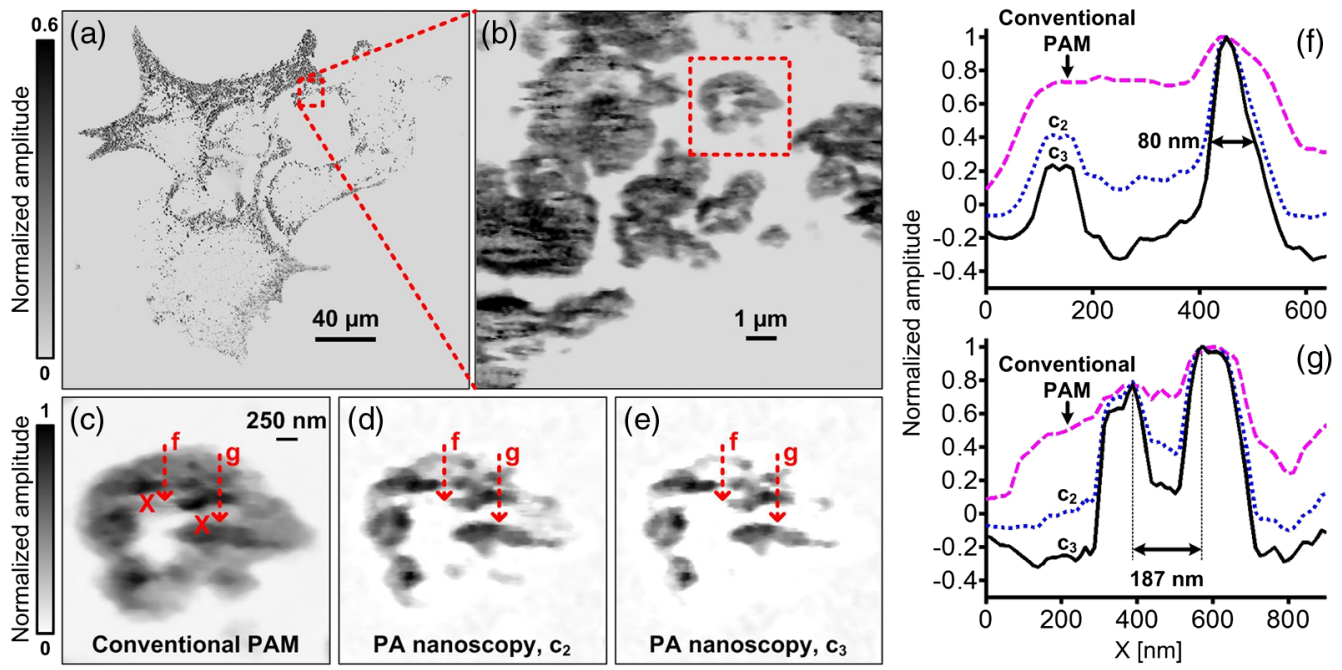

Fig. 5 Imaging melanoma cells. (a) and (b) Conventional PA images of melanoma cells. A cluster of melanosomes imaged by (c) conventional PAM, (d) second-order $\left(c_{2}\right)$, and (e) third-order $\left(c_{3}\right)$ PA nanoscopy (Video 4, QuickTime, $0.1 \mathrm{Mb}$ ) [URL: http://dx.doi.org/10.1117/1.JBO.19.8.086006.4]. (f) and (g) Normalized image amplitude along the dashed lines in (c) to (e). 


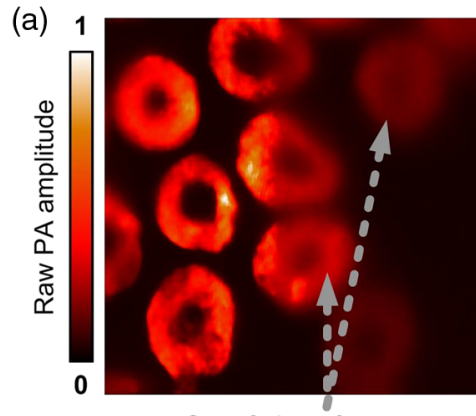

(b)

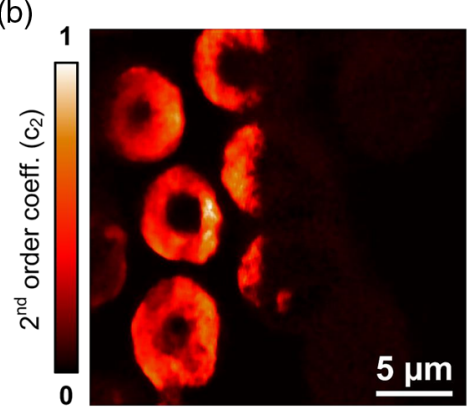

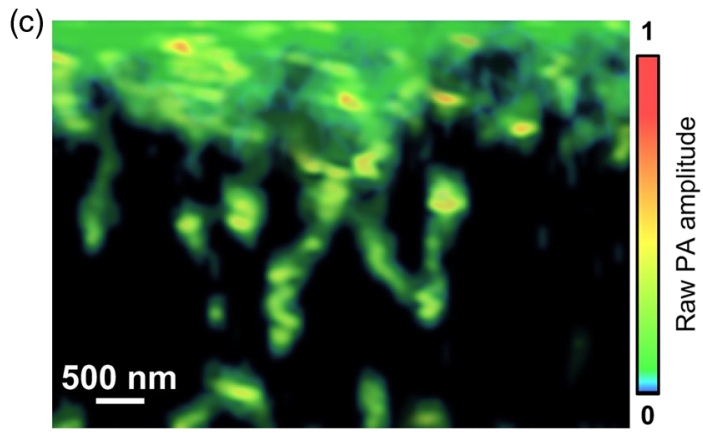

(d)

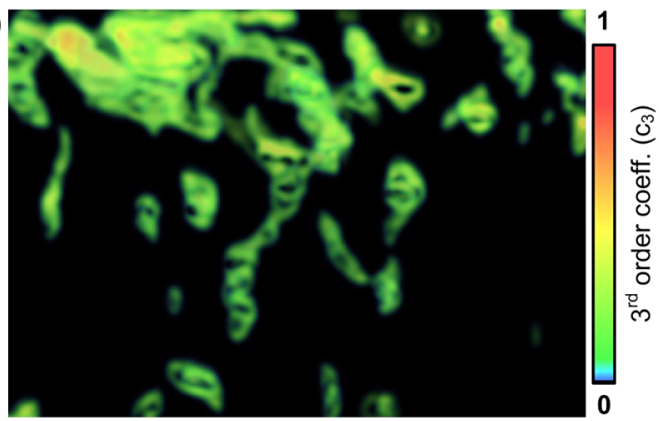

Fig. 6 Optical sectioning in PA nanoscopy. Cross-sectional images of red blood cells at $1 \mu \mathrm{m}$ depth acquired using a 1.2 NA objective by (a) conventional PAM and (b) second-order PA nanoscopy $\left(c_{2}\right)$. The high-order fluence dependence enables optical sectioning (Video 5, QuickTime, $0.4 \mathrm{Mb}$ [URL: http://dx.doi.org/10.1117/1.JBO.19.8.086006.5], Video 6, QuickTime, 2.5 Mb [URL: http:// dx.doi.org/10.1117/1.JBO.19.8.086006.6]). Three-dimensionally rendered structural images of mitochondria acquired at eight focal depths, $300 \mathrm{~nm}$ apart, by (c) conventional PAM and (d) third-order $\left(c_{3}\right)$ PA nanoscopy (Video 7, QuickTime, 0.9 Mb) [URL: http://dx.doi.org/10.1117/1.JBO.19.8.086006.7].
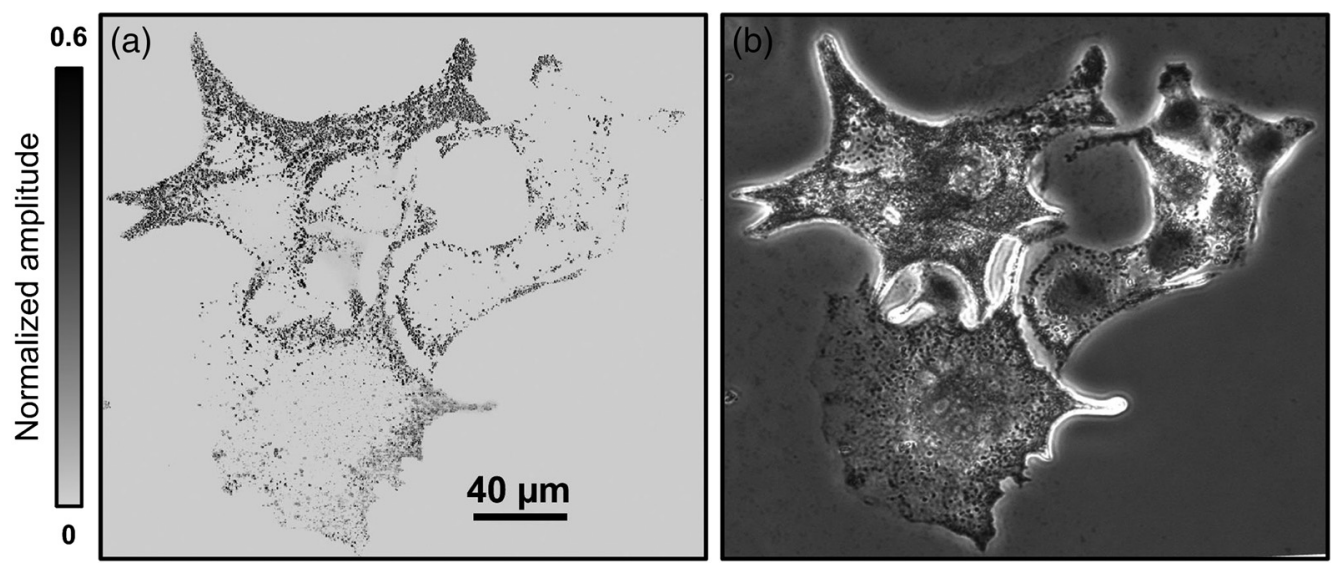

Fig. 7 Imaging melanoma cells before and after PA nanoscopy. (a) and (b) Melanoma cells imaged by (a) conventional PAM before PA nanoscopy and (b) phase microscopy after PA nanoscopy.

modalities, such as coherent anti-Stokes Raman scattering microscopy and super-resolution optical fluctuation imaging. ${ }^{33,34}$ The nonlinearity can be used to accentuate spatial gradients of molecule concentration. When quantification of molecule concentration is desired, the known relationship can be used to recover the absorption coefficient. Specificity in PA nanoscopy can be further improved using multiwavelength detection and spectral decomposition for different absorbers. ${ }^{27}$

The imaging speed can be significantly improved by increasing the pulse repetition rate of the laser (e.g., $100 \mathrm{kHz}$ ) or by employing multiple channels. ${ }^{35,36}$ In addition, existing fluorescence-based super-resolution techniques commonly use a picosecond or femtosecond laser, and a second laser is often required. By contrast, PA nanoscopy in its current embodiment relies only on a single nanosecond laser and an ultrasonic transducer, reducing the complexity and cost of the system. In summary, label-free PA nanoscopy of nonfluorescent chromophores greatly extends the capabilities of biological imaging.

\section{Acknowledgments}

We thank the Tissue Culture Support Center and Yu Shrike Zhang for culturing melanoma cells and fibroblasts. We thank Kate Nelson and Howard Wynder from the Nano Research Facility for technical help with atomic force microscopy and transmission electron microscopy imaging, and sample preparation. This work was sponsored in part by National Institutes of Health grants DP1 EB016986, R01 CA186567, R01 EB016963, R01 CA157277, and R01 CA159959. L.V.W. has a financial 
interest in Microphotoacoustics Inc. and Endra Inc., which, however, did not support this work. K.M. has a financial interest in Microphotoacoustics Inc.

\section{References}

1. B. Huang, M. Bates, and X. W. Zhuang, "Super-resolution fluorescence microscopy," Апnи. Rev. Biochem. 78(1), 993-1016 (2009).

2. V. Mueller et al., "STED nanoscopy reveals molecular details of cholesterol- and cytoskeleton-modulated lipid interactions in living cells," Biophys. J. 101(7), 1651-1660 (2011).

3. S. W. Hell and J. Wichmann, "Breaking the diffraction resolution limit by stimulated emission: stimulated-emission-depletion fluorescence microscopy," Opt. Lett. 19(11), 780-782 (1994).

4. S. W. Hell and M. Kroug, "Ground-state-depletion fluorescence microscopy - a concept for breaking the diffraction resolution limit," Appl. Phys. B 60(5), 495-497 (1995).

5. M. G. L. Gustafsson, "Nonlinear structured-illumination microscopy: wide-field fluorescence imaging with theoretically unlimited resolution," Proc. Natl. Acad. Sci. U. S. A. 102(37), 13081-13086 (2005).

6. E. Betzig et al., "Imaging intracellular fluorescent proteins at nanometer resolution," Science 313(5793), 1642-1645 (2006).

7. M. J. Rust, M. Bates, and X. W. Zhuang, "Sub-diffraction-limit imaging by stochastic optical reconstruction microscopy (STORM)," Nat. Methods 3(10), 793-795 (2006).

8. J. Bierwagen et al., "Far-field autofluorescence nanoscopy," Nano Lett. 10(10), 4249-4252 (2010).

9. Y. Cotte et al., "Marker-free phase nanoscopy," Nat. Photonics 7(2), 113-117 (2013).

10. D. A. Nedosekin et al., "Super-resolution nonlinear photothermal microscopy," Small 10(1), 135-142 (2014).

11. L. V. Wang and S. Hu, "Photoacoustic tomography: in vivo imaging from organelles to organs," Science 335(6075), 1458-1462 (2012).

12. L. V. Wang, "Multiscale photoacoustic microscopy and computed tomography," Nat. Photonics 3(9), 503-509 (2009).

13. L. V. Wang, "Tutorial on photoacoustic microscopy and computed tomography," IEEE J. Sel. Topics Quantum Electron. 14(1), 171-179 (2008).

14. V. P. Zharov, "Ultrasharp nonlinear photothermal and photoacoustic resonances and holes beyond the spectral limit," Nat. Photonics 5(2), 110-116 (2011).

15. A. Danielli et al., "Picosecond absorption relaxation measured with nanosecond laser photoacoustics," Appl. Phys. Lett. 97(16), 163701 (2010).

16. A. C. Tam and C. K. N. Patel, "2-photon absorption-spectra and crosssection measurements in liquids," Nature 280(5720), 304-306 (1979).

17. A. Danielli et al., "Single-wavelength functional photoacoustic microscopy in biological tissue," Opt. Lett. 36(5), 769-771 (2011).

18. J. Yao et al., "Photoimprint photoacoustic microscopy for three-dimensional label-free subdiffraction imaging," Phys. Rev. Lett. 112(1), 014302 (2014).

19. L. Gao et al., "Photothermal bleaching in time-lapse photoacoustic microscopy," J. Biophotonics 6(6-7), 543-548 (2013).

20. I. G. Calasso, W. Craig, and G. J. Diebold, "Photoacoustic point source," Phys. Rev. Lett. 86(16), 3550-3553 (2001).

21. S. Y. Xu et al., "Thermal expansion of confined water," Langmuir 25(9), 5076-5083 (2009).

22. L. V. Wang and H.-i. Wu, Biomedical Optics: Principles and Imaging, Wiley-Interscience, Hoboken, New Jersey (2007).

23. A. E. Siegman, Lasers, University Science Books, Mill Valley, California (1986).

24. B. Rao et al., "Real-time four-dimensional optical-resolution photoacoustic microscopy with $\mathrm{Au}$ nanoparticle-assisted subdiffractionlimit resolution," Opt. Lett. 36(7), 1137-1139 (2011).

25. D. A. Nedosekin et al., "Photothermal confocal spectromicroscopy of multiple cellular chromophores and fluorophores," Biophys. J. 102(3), 672-681 (2012).

26. C. Consani et al., "Energy transfer and relaxation mechanisms in cytochrome c," Chem. Phys. 396(1), 108-115 (2012).

27. C. Zhang et al., "Label-free photoacoustic microscopy of cytochromes," J. Biomed. Opt. 18(2), 20504 (2013).
28. A. V. Brusnichkin et al., "Ultrasensitive label-free photothermal imaging, spectral identification, and quantification of cytochrome $\mathrm{c}$ in mitochondria, live cells, and solutions," J. Biophotonics 3(12), 791-806 (2010).

29. H. C. Chen et al., "Mitofusins Mfn1 and Mfn2 coordinately regulate mitochondrial fusion and are essential for embryonic development," J. Cell Biol. 160(2), 189-200 (2003).

30. W. Denk, J. H. Strickler, and W. W. Webb, "Two-photon laser scanning fluorescence microscopy,” Science 248(4951), 73-76 (1990).

31. D. O. Lapotko and V. P. Zharov, "Spectral evaluation of laser-induced cell damage with photothermal microscopy," Lasers Surg. Med. 36(1), 22-30 (2005).

32. F. Fanjul-Velez et al., "Thermal damage analysis in biological tissues under optical irradiation: application to the skin," Int. J. Thermophys. 30(4), 1423-1437 (2009).

33. C. L. Evans and X. S. Xie, "Coherent anti-stokes raman scattering microscopy: chemical imaging for biology and medicine," Аппи. Rev. Anal. Chem. 1(1), 883-909 (2008).

34. T. Dertinger et al., "Fast, background-free, 3D super-resolution optical fluctuation imaging (SOFI), Proc. Natl. Acad. Sci. U. S. A. 106(52), 22287-22292 (2009).

35. L. A. Song, K. Maslov, and L. V. Wang, "Multifocal optical-resolution photoacoustic microscopy in vivo," Opt. Lett. 36(7), 1236-1238 (2011).

36. J. J. Yao et al., "Wide-field fast-scanning photoacoustic microscopy based on a water-immersible MEMS scanning mirror," J. Biomed. Opt. 17(8), 080505 (2012).

Amos Danielli is a senior lecturer at the Faculty of Engineering in Bar Ilan University, Israel. In 1997, 2002, and 2010, he received his BSc, $\mathrm{MSc}$, and PhD, respectively, in electrical engineering from Tel-Aviv University. In 2009 to 2014, he worked as a postdoctoral researcher in the Biomedical Engineering Department at Washington University in St. Louis. He is a three-time entrepreneur with 15 years of experience in executive $R \& D$ positions and in the development of multidisciplinary systems.

Konstantin Maslov graduated from Moscow Institute of Physics and Technology with a major in biophysics and received his $\mathrm{PhD}$ in physical acoustics from Moscow State University, Russia. After graduation, he worked in the Institute of Chemical Physics, Russian Academy of Sciences, and Texas A\&M University. Currently, he is a research associate professor in the Biomedical Engineering Department at Washington University in St. Louis, Missouri. His area of interest includes optical, photoacoustic, and acoustic imaging, and photoacoustic spectroscopy.

Alejandro Garcia-Uribe received his MSc and PhD degrees in electrical engineering from the Department of Electrical and Computer Engineering, Texas A\&M University, College Station, Texas. He is currently a researcher scientist at the Optical Imaging Laboratory, Department of Biomedical Engineering, Washington University in St. Louis. His research interests include biomedical optics, biomedical image analysis, microsensors, and digital signal processing.

Amy M. Winkler has a $\mathrm{PhD}$ in optical sciences from the College of Optical Sciences at the University of Arizona. During graduate school, she built tools for studying colorectal cancer in mouse models, under the guidance of Jennifer Barton. After graduate school, she analyzed the molecular sensitivity of photoacoustics while working as a postdoctoral researcher advised by Lihong Wang at Washington University in St. Louis. She currently works for Authentix, Inc. in Dallas, Texas.

Chiye Li received his BS in life sciences in 2007 from the University of Science and Technology of China in Hefei, China. He is currently a PhD student in biomedical engineering at Washington University in St. Louis. His research interests involve the application of photoacoustic imaging techniques in biological and medical studies.

Lidai Wang received his BASc and MASc degrees in instrument science and technology from Tsinghua University, Beijing, China, and received his $\mathrm{PhD}$ degree in mechanical engineering from the University of Toronto, Ontario, Canada. His PhD work was on automated microassembly and microrobotics. He is currently working on 
photoacoustic imaging in biomedical engineering at Washington University in St. Louis, Missouri.

Gerald W. Dorn II is the inaugural Philip and Sima K. Needleman professor and the associate chair (internal medicine) for translational research at Washington University in St. Louis. He received his medical school, internal medicine, and interventional cardiology training at the Medical University of South Carolina in Charleston, South Carolina. The Dorn laboratory investigates multiple aspects of genetic reprogramming in heart failure, such as protein kinase signaling and mitochondrial mechanisms of programmed cell death.
Lihong V. Wang is the Beare distinguished professor at Washington University. His book titled Biomedical Optics won the Goodman Award. He has published 395 journal articles with an $h$-index of 88 (>30,000 citations) and delivered 385 keynote/plenary/invited talks. His laboratory published first functional photoacoustic CT and three-dimensional photoacoustic microscopy. He serves as the editor-in-chief of the Journal of Biomedical Optics. He was awarded OSA's C.E.K. Mees Medal, NIH Director's Pioneer Award, and IEEE's Biomedical Engineering Award.

Yun Chen: biography is not available. 